\title{
Patient-Reported Outcomes in Eosinophilic Esophagitis and Achalasia
}

\author{
Alain Schoepfer, $M D^{1, *}$ \\ Alex Straumann, $M D^{2}$ \\ Ekaterina Safroneeva, $P h D^{3}$
}

\author{
Address \\ *,1 Division of Gastroenterology and Hepatology, Centre Hospitalier Universitaire \\ Vaudois, Rue de Bugnon 44,07/2409, 1011, Lausanne, Switzerland \\ Email: alain.schoepfer@chuv.ch \\ ${ }^{2}$ Swiss EoE Clinic, Praxis Römerhof, Olten, Switzerland \\ ${ }^{3}$ Institute of Social and Preventive Medicine, University of Bern, Bern, Switzerland
}

Keywords Achalasia - Eosinophilic esophagitis - Clinician-reported outcome · Patient-reported outcome •

Instrument $\cdot$ Questionnaire $\cdot$ Symptoms $\cdot$ Quality of life

\section{Opinion Statement}

The activity of a particular esophageal disease, such as eosinophilic esophagitis (EoE) or achalasia, can be evaluated using clinician-reported outcome (ClinRO) measures assessing various endoscopic, histologic, functional, and laboratory findings, and patient-reported outcome (PRO) measures. The patient-reported outcome (PRO) measures are those that are designed to be self-reported by patients. Commonly used PRO instruments include those that assess symptom severity, health-related quality of life, "general" quality of life, or health status. Regulatory authorities increasingly rely on PRO measures to support labeling claims for drug development. Validated PRO measures for various esophageal diseases are needed in order to unify and standardize the way disease activity is assessed, define clinically meaningful endpoints for use in interventional and observational studies, compare the efficacy/effectiveness of various therapies, and optimize therapeutic algorithms for management of these diseases. This article reviews commonly used PRO instruments designed to assess symptom severity and quality of life in adult patients with EoE and achalasia. 
How to measure the activity of esophageal diseases The activity of a particular esophageal disease can be assessed by the means of patient-reported outcome (PRO) and clinician-reported outcome (ClinRO) measures. The concept is depicted in Fig. 1.

The PRO is an "umbrella term" that, among other things, encompasses various measures of symptom severity, functioning (disability), health status, healthspecific quality of life, "general" quality of life, and general health perceptions. PRO instruments assess either a single underlying characteristic of a disease, in which case these instruments are termed unidimensional, or multiple constructs, in which case the instruments are termed multidimensional. Measures of symptom severity focus on a particular or several disease-related impairments. Measures of disability assess the degree of impairment that manifests itself when performing various daily activities, such as personal care, and locomotion. Health-specific quality of life measures tend to be multidimensional instruments that assess various aspects related to impairment and/or disability inherent to a particular disease. These instruments allow the users to stratify the subjects into various "health status" or "clinical states" based on responses to the items that query relevant information on patients' perception of health. As such, these instruments enable the comparisons between groups of patients with a particular disease that are treated by different therapeutic regimens and allow for monitoring of changes in "health status" or "clinical state" of a given patient [1]. In contrast, the "general" quality of life instruments evaluate the

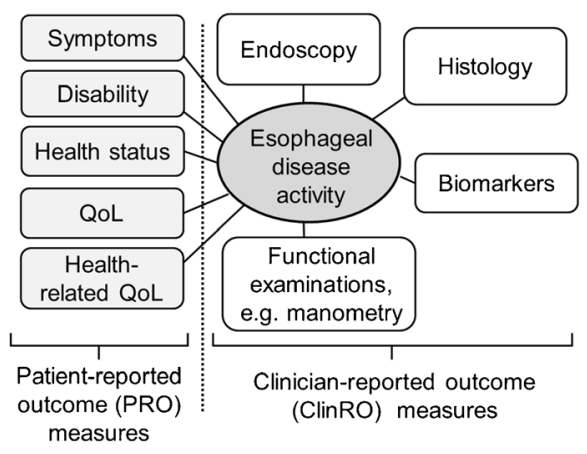

Fig. 1. Assessing activity of esophageal disease by patientreported outcome (PRO) or clinician-reported outcome (ClinRO) measures. QoL quality of life. patients' ability to fulfill their needs and assess the impact of a restriction or impairment on emotional wellbeing. These instruments help researchers to compare across different conditions and populations [1].

In esophageal diseases, endoscopic, histologic, and laboratory findings, as well as other aspects of gastrointestinal physiology, such as esophageal motility (as assessed by manometry) or gastric acid reflux (as assessed by impedance-pH-metric study), are evaluated using ClinRO measures.

The development and validation of PRO instruments is an iterative process that has been described in a position paper by the US Food and Drug Administration (US FDA) [2]. In the first phase, items are generated using patient input. The generated items are incorporated into an instrument (questionnaire), which is evaluated in a first and then validated in a second patient group. During the entire process, patient feedback is sought on numerous occasions. The instrument should be content valid, which means that it measures the diseaseassociated dimensions relevant to the patients, and responsive to change in a health status. The US FDA and the European Medicines Agency (EMA) put a particular emphasis on the use of validated PRO instruments in the trials of medical products in order to support labelling claims [2]. Interestingly, the EMA more frequently grants PRO label claims when compared to the US FDA [3].

Why do we need validated PRO instruments for assessment of the activity of esophageal diseases? First, these instruments help the users to standardize the language of disease activity assessment. Second, such validated PRO instruments and respective scores serve as endpoints in clinical trials and observational studies. The use of these standardized measures facilitates the comparison of the efficacy/effectiveness of various therapies and aids in optimization of therapeutic algorithms used to identify the appropriate therapy for patients.

Should the activity of esophageal diseases mainly be measured based solely on PRO, or ClinRO measures, or a combination of both? When assessing activity of any disease, it is important to take into account PRO, so long the disease-inherent alterations lead to generation of symptoms and changes in psychosocial functioning that can be reliably assessed by PRO measures. Similarly, it is important to take into account ClinRO measures, if these assess biologic findings that may serve as 
biomarkers for estimation of disease severity. We have recently described this concept elsewhere [4]. In esophageal diseases, such as EoE or GERD or achalasia, both PRO and ClinRO measures should be assessed to get a complete picture on disease activity overall.

In this review, the currently available PRO instruments assessing symptom severity and quality of life in adults with EoE and achalasia are discussed. A systematic review of PRO instruments for assessment of gastroesophageal reflux disease (GERD) activity has recently been published $[5 \bullet \bullet]$.

\section{PRO instruments for use in eosinophilic esophagitis} patients

Eosinophilic esophagitis (EoE) emerged as distinct disease in the early 1990 s $[6 \bullet, 7 \bullet]$. In industrialized countries, an incidence rate of about 1 new patient per 10,000 inhabitants per year and prevalence rate of approximately 1 patient per 2000 inhabitants is observed [8-11]. In the consensus guidelines first published in 2007 and updated in 2011, the experts defined EoE as "a chronic, immune/antigen-mediated, esophageal disease, characterized clinically by symptoms related to esophageal dysfunction and histologically by eosinophil-

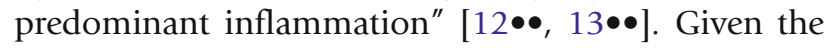
fact that esophageal eosinophilia can be caused by other diseases, such as GERD, Crohn's disease, or achalasia, these should be excluded prior to establishing the EoE diagnosis. Historically, different non-EoE-specific or nonvalidated instruments have been used in clinical trials and observational studies. As a consequence, relationship between EoE symptom severity and histologic activity was a matter of much controversy, as dissociation between severity symptoms and histologic findings have been described in some [14-16], but not other studies $[17,18]$. The validated instruments for assessment of the severity of various EoE-specific symptoms and biologic findings as well as EoE-specific quality of life have recently become available.

When assessing symptom severity in EoE patients, one has to keep in mind a few particularities. First, EoE symptom presentation is age-dependent [19]. Feeding difficulties and failure to thrive are symptoms more typical of infants and toddlers, while vomiting and abdominal pain are symptoms more characteristic of school-aged children. Dysphagia and food impactions are principal symptoms of adolescent and adult EoE patients. Second, patients need to recall their symptoms over a defined time period. Although regulatory authorities emphasize the importance of using daily electronic diaries in the context of clinical trials sponsored by pharmaceutical companies, the use of these diaries may not be entirely feasible in the context of long-term observational studies. As such, other recall periods must be considered. Third, the severity of dysphagia and behavioral adaptations to living with dysphagia may vary depending on the consistency of foods consumed by the patients. Therefore, instrument developers should attempt to capture the full spectrum of various behavioral adaptations and dysphagia severity by separately examining these for each distinct food consistency.

An overview of the available PRO instruments for use in adult EoE patients is provided below.

\section{EoE activity index (EEsAI) PRO instrument}

In 2014, Schoepfer et al. described the development and validation of the EoE activity index (EEsAI) PRO instrument (copyrighted by the University of Bern, Switzerland) $[20 \bullet \bullet$. The work was carried out by the international EEsAI study group in line with the recommendations of the US FDA [2]. Multivariable linear regression analysis and analysis of variance with patient global assessment as dependent variable and distinct symptoms as independent variables were used to develop a PRO score by quantifying the extent to which symptoms explained the variability in patient global assessment. The EEsAI PRO instrument was evaluated in a group of 153 adult EoE patient and validated in the group of another 120 patients. The EEsAI PRO score of 0 indicates lack of EoE-specific symptoms, while a score of 100 points indicates most severe symptoms. The EEsAI PRO instrument consists of the five following components: frequency of dysphagia episodes, duration of dysphagia episodes, pain when swallowing, severity of dysphagia occurring when eating foods of various consistencies, and behavioral adaptations, such as avoiding distinct food due to EoE or cutting food into small pieces. A median of $8 \mathrm{~min}$ is required for instrument completion. Patients deemed the instrument as easy to complete and capturing most of their EoE-

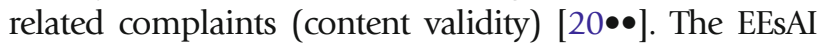
PRO instrument is available with a symptom recall period of 7 days and 24 hours. The responsiveness of the instrument is currently evaluated in a number of clinical trials.

\section{Dysphagia symptom questionnaire}

In 2013, the development of the dysphagia symptom questionnaire (DSQ) carried out in collaboration with 
Meritage Pharma, Inc. (copyright holder) was published by Dellon et al. [21••]. The DSQ is an electronic daily diary (symptom recall period of $24 \mathrm{~h}$ ) consisting of three items that report on solid-food-avoidance days, dysphagia days, and actions taken to resolve dysphagia episode. At the time of the publication, the food described as solid was not actually specified. The scoring algorithm is expert-based. A score of 0 indicates lack of dysphagia, while a score of 5 points indicates severe dysphagia. The field trial was completed by adult $(n=18)$ and adolescent $(n=17)$ EoE patients. The DSQ score has strong positive association with the number of dysphagia days $(R=0.96$, $P<0.001)$. The instrument was shown to be contentvalid and is currently evaluated in a number of clinical trials (personal communication).

\section{Straumann dysphagia index instrument}

Nonvalidated, the EoE-specific Straumann dysphagia index (SDI) instrument for adults with EoE was first used to assess symptom severity in a randomized, placebo-controlled trial evaluating the efficacy of swallowed topical corticosteroids [22]. The measure and the scoring algorithm (score ranges from 0 to 9 points) were developed by a physician (AS). The SDI assesses frequency and intensity of dysphagia events; neither dysphagia caused by foods of various consistencies nor behavioral adaptations to living with dysphagia are evaluated. Responsiveness, defined as a decrease in 3 or more points from the baseline score, was demonstrated in this trial [22].

\section{Mayo dysphagia questionnaire}

Grudell and coworkers published the paper on the development and validation of the Mayo dysphagia questionnaire (MDQ) [23]. The MDQ is designed to evaluate dysphagia severity in adult patients affected by different esophageal diseases. This non-EoE-specific PRO instrument consists of the 28 items that query the following: frequency and severity of dysphagia (17 items), concomitant allergies ( 3 items), the use of acid-suppressive medication ( 3 items), past esophageal surgery ( 2 items), presence of GERD ( 1 item), past esophageal dilations ( 1 item), and overall duration of trouble swallowing (1 item). The MDQ evaluates dysphagia severity and behavioral adaptations to living with dysphagia (food modification and time to eat a regular meal) in the context of consuming foods of different consistencies. The MDQ, 30-day version (a 30-day recall period), has shown to be reliable and was validated in a large group of patients experiencing dysphagia [24]. On average, patients take $10 \mathrm{~min}$ to complete the MDQ, 30-day version. The instrument is also available with a recall period of 2 weeks (MDQ, 2-week version) [14].

\section{Instruments for assessment of quality of life in adult EoE patients}

\section{Adult EoE quality of life instrument}

In 2011, Taft and coworkers published a paper describing the development of EoE-specific quality of life measure for adults (EoO-QOL-A; copyrighted by Northwestern University, Chicago, IL, USA) [25••]. Two hundred and one patients between ages of 18 and 70 years old were enrolled into the study. By the use of qualitative methods, authors examined the whole spectrum of the EoE-specific concerns and their impact on quality of life. The 37-item instrument is split into the five following subscales: eating/diet impact, social impact, emotional impact, disease anxiety, and choking anxiety. Patients that do not undergo dietary therapy complete only 32 items. Authors chose a recall period of 7 days. The EoOQOL-A instrument was shown to be reliable (internal consistency, split-half, and test-retest). The QoL score closely correlated with symptom severity score as assessed by the adult PRO instrument $(r=0.610$, $p<0.001)$ [26].

\section{PRO instruments for gastroesophageal reflux disease}

An excellent systematic review on this topic has been published by Vakil and coworkers in 2013 [5••]. Authors identified existing PRO instruments for assessment of severity gastroesophageal reflux disease (GERD) symptoms and evaluated whether these instruments have been developed in accordance with regulatory requirements of US FDA and EMA (e.g., have been shown to be 
reliable [content, construct and test-retest, internal consistency], and responsive to change) [27]. Of the identified instruments, 15 PRO instruments underwent psychometric evaluation. Of these, eight instruments were developed to assess severity of GERD symptoms, two were developed to diagnose GERD, four PRO instruments were developed for both evaluation and diagnosis of GERD, and one PRO instrument was designed to screen for the existence of GERD. Developers of only five of the PRO instruments followed most steps outlined in regulatory guidance documents. These five instruments were also used as endpoint measures in clinical trials. Nonetheless, the authors concluded that, as of yet, none of the PRO instrument developed to date met all of the regulatory requirements for an outcome instrument in GERD trials [5••].

\section{PRO instruments for achalasia}

Achalasia is a rare disorder characterized by a loss of peristalsis and a failure of the lower esophageal sphincter (LES) relaxation with swallowing. An annual incidence of approximately 1.6 patients/100,000 inhabitants and a prevalence of 10 patients per 100,000 inhabitants are observed [28]. Affected patients most often suffer from dysphagia for solids and liquids and regurgitation of undigested food or saliva $[29,30 \bullet]$. A mean diagnostic delay (time interval from onset of first symptoms to diagnosis) of 4.7 years was documented in adult achalasia patients [31]. The PRO instruments used in achalasia are reviewed below.

\section{Instruments for assessment of symptoms in adult achalasia patients}

The most frequently used scores for clinical assessment in achalasia are the Eckardt score and the Vantrappen classification.

\section{Eckardt score}

The Eckardt score evaluates the frequency or severity of the following components: weight loss, dysphagia, retrosternal pain, and regurgitation [32, 33]. Each item ranks from 0 to 3 points with the final score ranking from 0 to 12 points. Clinical remission was defined as Eckardt score of 3 and less, persisting for at least 6 months [34]. The modification of the Eckardt score was also published. Specifically, chest pain was rescored on a scale from 0 to 4 (none, $<$ monthly, monthly, weekly, daily), such as the entire instrument scores up to a maximum of 13 points [29]. Using this modified scale for chest pain, a definition of clinical remission of 4 points or less points was proposed. The scoring algorithm is physician-based. For the development of the score no systematic patient input, as proposed by regulatory authorities, was used. The score is completed by the caregiver during a structured clinical interview. The score is also used for clinical staging of achalasia with stages from 0 (Eckardt score 0-1) to 3 (Eckardt score >6). A Korean group found no significant correlation between the Eckardt symptom score and Eckardt dysphagia grade and radiologic indices of achalasia severity [35]. The Eckardt symptom score significantly correlated with the esophagogastric junction distensibility as measured by the functional lumen imaging probe (FLIP ${ }^{\mathrm{TH}}$ ) [36]. 


\section{Vantrappen classification}

Similar to the Eckardt score, the Vantrappen classification is a physician-based instrument [37]. Patients are interviewed about the frequency of esophageal symptoms, such as dysphagia, regurgitation, and chest pain. Depending on whether these symptoms occur never, occasionally, more than once a week, or daily, a symptoms score on a scale of $0-3$ is applied. The total score ranges from 0 to 9 points. Clinical remission was arbitrarily defined as a score of $\leq 2$ and failure of treatment (or relapse) as a score of $\geq 3$ points. In a prospective trial randomizing 25 achalasia patients to undergo pneumatic dilation and 25 patients to undergo myotomy, patients were assessed clinically using the Eckardt score, the Vantrappen classification, as well as the Adam's stages [38]. Authors noted a significant correlation between the Vantrappen classification and the Eckardt score.

\section{Achalasia symptom questionnaire}

The achalasia symptom questionnaire (ASQ) was published in 2010. The ASQ is a 11-item measure designed to assess symptoms typical of achalasia [39]. For the first nine items, the symptoms severity is assigned a score between 0 (better) and 4 (worse). The symptoms severity queried in items 10 and 11 is assigned a score between 0 and 1 and 0 and 3, respectively. The total ASQ score of 0 indicates the lack of achalasia symptoms, while a score of 67 points indicates severe achalasia symptoms. Item selection and scoring algorithm are based on physician input. Using the data from the group of 124 adult patients, the authors developed a mathematical model to predict achalasia in order to fasttrack patient to manometric confirmation of the suspected diagnosis [39].

\section{Other clinical scores for achalasia}

Other physician-developed scores, such as the one described by Vaezi and colleagues, exist [40]. The authors performed interviews in patients. The total achalasia symptom score reaches a maximum of 15 points and is a sum of dysphagia, regurgitation, and chest pain subscores. The frequency of each symptom was graded on a scale from 0 to $5(0=$ none; $1=$ once per month or less; $2=$ once per week, up to 3-4 times a month; $3=2-4$ times per week; $4=$ once per day; $5=$ several times per day). The authors found that timed barium esophagogram better predicted the long-term success after pneumatic dilation than symptom assessment [40].

In summary, all available scoring systems to assess symptoms in achalasia were developed without psychometric evaluation and most of them are completed by caregivers during patient interviews. This stands in contrast to the current US FDA guidelines for PRO development [2]. Nevertheless, it is important to point out that these scores were developed well before comprehensive guidelines on PRO development and validation were published by regulatory authorities [2].

\section{Instruments used to assess quality of life in adult achalasia patients}

As mentioned previously, quality of life can be assessed either by health-related quality of life measures that are specific for a given disease or by generic quality of life. The following instruments exist for assessment of quality of life in adult achalasia patients. 


\section{Health-related quality of life score by Urbach}

The authors conducted semi-structured interviews with achalasia patients $(n=7)$ and sought expert input to create a list of items for development of disease-specific quality of life measure [41]. The instrument was evaluated in a group of 70 patients. A ten-item instrument covered the following domains: food tolerance, dysphagia-related behavioral modifications, pain, heartburn, distress, lifestyle limitation, and satisfaction. The total score is calculated by summing the score for each item (lowest score 10 points, highest score 31 points), where after these outcomes are transformed to a 0-100 point scale. A lower score represents a better health-related QoL. The instrument was reliable and showed construct validity; however, it was not validated in the initial form [41].

Frankhuisen et al. translated the measure developed by Urbach and coworkers into Dutch and validated it in a group of 171 achalasia patients [42]. The instrument proved to be easy in use. Authors concluded that this achalasia instrument was a reliable and valid measure for comparison of different groups of achalasia patients [42].

\section{Disease-specific health-related quality of life questionnaire for achalasia}

The disease-specific health-related quality of life questionnaire for esophageal achalasia (AE-18) is a self-administered measure that has been developed in a group of 104 patients and prospectively validated in a second group of adult achalasia patients. The qualitative methodology was published only in abstract form [43]. The instrument consists of 18 items encompassing the following four domains: physical functioning, psychological functioning, social functioning, and sleep. Patients were asked to provide the answers on a five-point Likert scale ranging from 1 ("always)" to 5 ("never"). Higher scores correspond to better quality of life. Scores for each domain are calculated as the median of the scores of corresponding items. The responsiveness of the AE-18 instrument was evaluated in 65 prospectively included adult achalasia patients, of whom 47 were dilated and 18 underwent laparoscopic Heller myotomy [44]. Following treatment, AE-18 global score and subscales scores increased; changes in quality of life were associated with improvement in symptoms.

\section{SF-36 ${ }^{\circledast}$ health survey}

The SF-36 health survey is a generic outcome measure designed to examine a person's perceived health status $[45,46]$. The selfadministered instrument takes 5-10 min to complete. It includes a multiitem scale that measures each of the following eight health concepts: (1) physical functioning, (2) role limitations because of physical health problems, (3) bodily pain, (4) social functioning, (5) general mental health (psychological distress and psychological well-being), (6) role limitations because of emotional problems, (7) vitality (energy/ fatigue), and (8) general health perceptions. Answers to each item are scored. For each health concept, these scores are summed to produce raw scale scores, which are then transformed to a 0-100 scale. Generic quality of life in achalasia patients was evaluated in several studies. Frankhuisen et al. found that many achalasia patients remain severely 


\section{Conclusions and outlook}

symptomatic after treatment and also suffer from decreased healthrelated quality of life [47]. Liu et al. evaluated health-related quality of life in 35 adult achalasia patients undergoing transoral endoscopic myotomy (POEM) and found that, besides the safety and efficacy of the procedure in the short-term run, it also relieved symptom severity and improved health-related quality of life [48].

The first guidelines on the development and validation of PRO, reflecting the opinion of regulatory authorities, were published in 2007. Before 2007, multiple instruments have been developed to assess symptom severity and quality of life in adult patients suffering from EoE and achalasia. It is clear that these instruments did not incorporate all recommendations from regulatory authorities that are currently regarded as mandatory to establish a labeling claim for the efficacy of a particular therapeutic intervention that is measured by a given PRO instrument. For EoE, there are currently two PRO instruments (the EEsAI PRO instrument and the Dysphagia Symptom Questionnaire [DSQ]) for assessment of symptom severity available that were developed according to the guidelines of regulatory authorities. In addition, the EoO-QOL-A instrument was developed following these guidelines and allows measurement of EoE-specific quality of life. In achalasia, there is currently no PRO instrument available that was developed according to the guidelines from regulatory authorities. Further collaborative efforts between different stakeholders are needed in order to define to what extent therapeutic success should be defined by either PRO or biologic measures.

\section{Acknowledgments}

This work was supported by a grant from The International Gastrointestinal Eosinophil ResearcherS (TIGERS) and the Swiss National Science Foundation (Swiss National Science Foundation, grant no. 32003B_160115/1 to AS).

\section{Compliance With Ethical Standards}

\section{Conflict of Interest}

Alain Schoepfer and Alex Straumann are among the copyright holders of the Eosinophilic Esophagitis Activity Index (EEsAI) patient-reported outcomes instrument.

Human and Animal Rights and Informed Consent

This article does not contain any studies with human or animal subjects performed by any of the authors. 
Papers of particular interest, published recently, have been highlighted as:

- Of importance

$\bullet \quad$ Of major importance

1. Guyatt GH, Feeny DH, Patrick DL. Measuring healthrelated quality of life. Ann Intern Med. 1993;118:622-9.

2. Patrick DL, Burke LB, Powers JH, Scott JA, Rock EP, Dawisha S, et al. Patient-reported outcomes to support medical product labeling claims: FDA Perspective. Value Health. 2007;10 Suppl 2:S125-37.

3. DeMuro C, Clark M, Doward L, Evans E, Mordin M, Gnanasakthy A. Assessment of PRO label claims granted by the FDA as compared to the EMA (20062010). Value Health. 2013;16:1150-5.

4. Schoepfer AM, Hirano I, Katzka DA. Eosinophilic esophagitis: overview of clinical management. Gastroenterol Clin North Am. 2014;43:329-44.

5.•• Vakil NB, Halling K, Becher A, Ryden A. Sytematic review of patient-reported outcome instruments for gastroesophageal reflux disease symptoms. Eur J Gastroenterol Hepatol. 2013;25:2-14.

The systematic review evaluated the existing the patient-reported outcome instruments in order to identify instruments that fulfill regulatory requirements and can be used in trials to support claims in medical product labeling.

6. Straumann A, Spichtin HP, Bernoulli R, Loosli J, Vögtlin J. Idiopathic eosinophilic esophagitis: a frequently overlooked disease with typical clinical aspects and discrete endoscopic findings. Schweiz Med Wochenschr. 1994;124:1419-29.

German. Second paper to describe EoE as distinct entity.

7. $\quad$ Attwood SE, Smyrk TC, Demeester TR, Jones JB. Esophageal eosinophilia with dysphagia. A distinct clinicopathological syndrome. Dig Dis Sci. 1993;38:109-16.

First paper to describe EoE as distinct entity.

8. Prasad GA, Alexander JA, Schleck CD, Zinsmeister AR, Smyrk TC, Elias RM, et al. Epidemiology of eosinophilic esophagitis over three decades in Olmsted County, Minnesota. Clin Gastroenterol Hepatol. 2009; 7:1055-61.

9. Hruz P, Straumann A, Bussmann C, Heer P, Simon HU, Zwahlen M, et al. Escalating incidence of eosinophilic esophagitis: a 20-year prospective, population-based study in Olten County, Switzerland. J Allergy Clin Immunol. 2011;128:1349-50.

10. Dellon ES, Jensen ET, Martin CF, Shaheen NJ, Kappelman MD. Prevalence of eosinophilic esophagitis in the United States. Clin Gastroenterol Hepatol. 2014;12:589-96.

11. Kapel RC, Miller JK, Torres C, Aksoy S, Lash R, Katzka DA. Eosinophilic esophagitis: a prevalent disease in the United States that affects all age groups. Gastroenterology. 2008;134:1316-21.
12.• Furuta GT, Liacouras CA, Collins MH, Gupta SK, Justinich C, Putnam PE, et al. Eosinophilic esophagitis in children and adults: a systematic review and consensus recommendations for diagnosis and treatment. Gastroenterology. 2007;133:1342-63.

First consensus definition of EoE.

$13 . \bullet \quad$ Liacouras CA, Furuta GT, Hirano I, Atkins D, Attwood SE, Bonis PA, et al. Eosinophilic esophagitis: updated consensus recommendations for children and adults. J Allergy Clin Immunol. 2011;128:3-20.

Updated consensus definition of EoE.

14. Alexander JA, Jung KW, Arora AS, Enders F, Katzka DA, Kephardt GM, et al. Swallowed fluticasone improves histologic but not symptomatic response of adults with eosinophilic esophagitis. Clin Gastroenterol Hepatol. 2012;10:742-9.

15. Pentiuk S, Putnam PE, Collins MH, Rothenberg ME. Dissociation between symptoms and histological severity in pediatric eosinophilic esophagitis. J Pediatr Gastroenterol Nutr. 2009;48:152-60.

16. Gupta SK, Vitanza JM, Collins MH. Efficacy and safety of oral budesonide suspension in pediatric patients with eosinophilic esophagitis. Clin Gastroenterol Hepatol. 2015;13:66-76.

17. Straumann A, Conus S, Degen L, Felder S, Kummer M, Engel $\mathrm{H}$, et al. Budesonide is effective in adolescent and adult patients with active eosinophilic esophagitis. Gastroenterology. 2010;139:1526-37.

18. Dohil R, Newbury R, Fox L, Bastian J, Aceves S. Oral viscous budesonide is effective in children with eosinophilic esophagitis in a randomized, placebocontrolled trial. Gastroenterology. 2010;139:418-29.

19. Noel RJ, Putnam PE, Rothenberg ME. Eosinophilic esophagitis. N Engl J Med. 2004;351:940-1.

20.• Schoepfer A, Straumann A, Panczak R, Coslovsky M, Kuehni CE, Maurer E, et al. Development and validation of a symptom-based activity index for adults with eosinophilic esophagitis. Gastroenterology. 2014;147:1255-66.

One of the validated instruments for assessment of symptom severity in adult patients with EoE.

21.• Dellon ES, Irani AM, Hill MR, Hirano I. Development and field-testing of a novel patient-reported outcome measure of dysphagia in patients with eosinophilic esophagitis. Aliment Pharmacol Ther. 2013;38:634-42.

One of the validated instruments for assessment of symptom severity in adult patients with EoE.

22. Straumann A, Spichtin HP, Grize L, Bucher KA, Beglinger C, Simon HU. Natural history of primary eosinophilic esophagitis: a follow-up of 30 adult 
patients for up to 11.5 years. Gastroenterology. 2003;125:1660-9.

23. Grudell AB, Alexander JA, Enders FB, Pacifico R, Fredericksen M, Wise JL, et al. Validation of the Mayo Dysphagia Questionnaire. Dis Esophagus. 2007;20:202-5.

24. McElhiney J, Lohse MR, Arora AS, Peloquin JM, Geno DM, Kuntz MM, et al. The Mayo Dysphagia Questionnaire-30: documentation of reliability and validity of a tool for intervential trials in adults with esophageal disease. Dysphia. 2010;25:221-30.

$25 . \bullet$ Taft TH, Kern E, Kwiatek MA, Hirano I, Gonsalves N, Keefer L. The adult eosinophilic esophagitis quality of life questionnaire: a new measure of health-related quality of life. Aliment Pharmacol Ther. 2011;34:790-8.

Validated instrument for assessment of quality of life in adult patients with EoE.

26. Safroneeva E, Coslovsky M, Kuehni CE, Zwahlen M, Haas NA, Panczak R, et al. Eosinophilic oesophagitis: relationship of quality of life with clinical, endoscopic, and histological activity. Aliment Pharm Ther. 2015;42:1000-10.

27. EMA. Guideline on the evaluation of drugs for the treatment of gastroesophageal reflux disease. London, UK: Committee for Medicinal Products for Human Use of the European Medicines Agency;2009. Available at: http://www.ema.europa.eu/docs/en_GB/document library/Scientific_guideline/2010/01/WC500052741. pdf (accessed October 21st 2015).

28. Sadowski DC, Ackah F, Jiang B, Svenson LW. Achalasia: incidence, prevalence and survival. A population-based story. Neurogastroenterol Motil. 2010;22:e256-61.

29. Eckardt VD, Stauf B, Bernhard B. Chest pain in achalasia: patient characteristics and clinical course. Gastroenterology. 1999;116:1300-4.

30. Vaezi MF, Pandolfino JE, Vela MF. ACG clinical guideline: diagnosis and management of achalasia. Am J Gastroenterol. 2013;108:1238-49.

Guidelines on achalasia.

31. Eckardt VF, Kohne U, Junginger T, Westermeier T. Risk factors for diagnostic delay in achalasia. Dig Dis Sci. 1997;42:580-5.

32. Eckardt VF, Aignherr C, Bernhard G. Predictors of outcome in patients with achalasia treated by pneumatic dilation. Gastroenterology. 1992;103:1732-8.

33. Eckardt VF. Clinical presentation and complications of achalasia. Gastrointest Endosc Clin N Am. 2001;11:281-92.

34. Gockel I, Junginger T, Bernhard G, Eckardt VF. Heller myotomy for failed pneumatic dilation in achalasia. How effective is it? Ann Surg. 2004;239:371-7.

35. Moon JT, Jung IS, Kim YS, Cho SH, Park H, Lee SI. Correlation between clinical symptoms and radiologic findings before and after pneumatic balloon dilation for achalasia. Korean J Gastroenterol. 2008;52:16-20.
36. Pandolfino JE, de Ruigh A, Nicodème F, Xiao Y, Boris L, Kahrilas PJ. Distensibility of the esophagogastric junction assessed with the functional lumen imaging probe $\left(\mathrm{FLIP}^{\mathrm{TM}}\right)$ in achalasia patients. Neurogastroenterol Motil. 2013;25:496-501.

37. Annese V, Basciani M, Perri F, Lombardi G, Frusciante $\mathrm{V}$, Simone $\mathrm{P}$, et al. Controlled trial of botulinum toxin injection versus placebo and pneumatic dilation in achalasia. Gastroenterology. 1996;111:1418-24.

38. Gockel I, Junginger T. The value of scoring achalasia: a comparison of current systems and the impact of tratment - the surgeon's viewpoint. Am Surg. 2007;73:327-31.

39. Ferri LE, Cools-Lartigue J, Cao J, Miller L, Mayrand S, Fried GM, et al. Clinical predictors of achalasia. Dis Esophagus. 2010;23:76-81.

40. Vaezi MF, Baker ME, Achkar E, Richter JE. Timed barium esophagogram: better predictor of long term success after pneumatic dilation in achalasia than symptom assessment. Gut. 2002;50:765-70.

41. Urbach DR, Tomlinson GA, Harnish JL, Martino R, Diamant NE. A measure of disease-specific health-related quality of life for achalasia. Am J Gastroenterol. 2005; 100:1668-76.

42. Frankhuisen R, Heijkoop R, van Herwaarden AM, de Vries DR, Smout AJ, Baron A, et al. Validation of a disease-specific quality of life-questionnaire in a large sample of Dutch achalasia patients. Dis Esophagus. 2008;21:544-50.

43. Ortiz V, Garrigues V, Casanova C, et al. Development and validation of a disease-specific health-related quality of life (HRQL) questionnaire for esophageal achalasia (EA). Gastroenterology. 2009;136:A-382.

44. Garrigues V, Ortiz V, Casanova C, Bujanda L, MorenoOsset E, Rodriguez-Tellez M, et al. Disease-specific health-related quality of life in patients with esophageal achalasia before and after therapy.

Neurgastroenterol Motil. 2010;22:739-45.

45. Ware Jr JE, Sherbourne CD. The MOS 36 item short form health survey (SF 36). 1. Conceptual framework and item selection. Med Care. 1992;30:473-83.

46. McHorney CA, Ware Jr JE, Raczek AE. The MOS 36 item short form health survey (SF 36). 2. Psychometric and clinical tests of validity measuring physical and mental health constructs. Med Care. 1993;31:247-63.

47. Frankhuisen R, van Heerwaarden MA, Heijkoop R, Smout AJ, Baron A, Vermeijden JR, et al. Persisting symptoms and decreased health-related quality-of-life in a cross-sectional study of treated achalasia patients. Aliment Pharmacol Ther. 2007;26:899-904.

48. Liu XJ, Tan YY, Yang RQ, Duan TY, Zhou JF, Zhou XL, et al. The outcomes and quality of life of patients with achalasia after peroral endoscopic myotomy in the short-term. Ann Thorac Cardiovasc Surg. 2015;21:507-12. 